\title{
A Fisioterapia Digital em Oncoginecologia durante a Pandemia de Covid-19
}

doi: https://doi.org/10.32635/2176-9745.RBC.2020v66nTemaAtual.1082

\author{
Digital Physical Therapy in Gynecologic Oncology during the COVID-19 Pandemic \\ La Fisioterapia Digital en Oncoginecología durante Pandemia de Covid-19
}

Raquel Boechat de Moura Carvalho'; Kamila Rodrigues Ferreira²; Felipe Cardozo Modesto ${ }^{3}$

\section{INTRODUÇÃO}

A nova doença denominada Covid-19 (do inglês, coronavirus disease 2019) é uma preocupação em termos de saúde em nível global. Reconhecida em dezembro de 2019, rapidamente demonstrou estar relacionada à síndrome respiratória aguda grave do coronavírus 2 (do inglês, severe acute respiratory syndrome coronavirus 2 Sars-CoV-2). O surto de Covid-19 apresentou desafios críticos para a saúde pública, pesquisa e comunidades médicas ${ }^{1}$. Sendo necessários esforços consideráveis para reduzir a transmissão ${ }^{2}$.

Os tratamentos oncológicos, como a quimioterapia e a cirurgia, levam os pacientes à imunossupressão, deixando-os mais suscetíveis a pneumonias, em razão da resposta imunológica enfraquecida por bactérias e vírus respiratórios. Até o momento, não há diretrizes bem estabelecidas para pacientes com câncer, mas é sabido que apresentam maior risco de doença grave e morte nos casos de Covid-193,4.

Prestar atendimento a pacientes com câncer, em meio a essa pandemia, tem sido extremamente desafiador, dado seu status vulnerável e a natureza agressiva de sua doença subjacente. Os centros de câncer têm aderido a diretrizes rígidas de controle de infecçóes, em ambiente hospitalar e ambulatorial, incluindo a reduçáo de circulaçáo de pessoas em suas unidades ${ }^{5,6}$.

Medidas restritivas sobre interrupção do fluxo entre cidades, limitações de deslocamento em transportes públicos, medidas preventivas de distanciamento social com conscientização da população para permanecer em casa, e orientaçóes sobre busca de assistência à saúde em casos essenciais têm sido recomendadas e as visitas ambulatoriais foram reduzidas ${ }^{6}$. Essas instruções têm sido implementadas de modo gradual e distintas nos diferentes aspectos socioeconômicos, culturais, de características dos sistemas políticos e de saúde, bem como dos procedimentos operacionais na sua implementação ${ }^{7}$.

No desafio do controle do câncer, mulheres com neoplasias ginecológicas em países de média e baixa rendas são mais afetadas na incidência e na mortalidade do câncer, comparados aos países desenvolvidos. A oncoginecologia se concentra nos cânceres do sistema reprodutor feminino, incluindo câncer do colo do útero, ovários, corpo do útero (endométrio), trompas de falópio, vagina e vulva. Dada a atual situação em evolução, são necessárias abordagens pragmáticas para lidar com os desafios de minimizar efeitos adversos do tratamento e do avanço da doença que deixam sequelas funcionais muito limitantes, que impactam diretamente no bem-estar físico e psicossocial dessas mulheres ${ }^{8}$. Todos esses fatores contribuem para dificultar o acesso dessas pacientes aos serviços de fisioterapia não adiáveis.

Dentro desse contexto, a fisioterapia digital surge como um recurso para os cuidados a distância, ajudando a romper as barreiras encontradas na prática do atendimento presencial. Suas primeiras iniciativas surgiram em 2017, em uma colaboração conjunta da World Confederation for Physical Therapy (WCPT) e do International Network of Physiotherapy Regulatory Authorities (INPTRA).

Em 2020, com as restrições vigentes da pandemia mundial de Covid-19, ficam autorizadas as práticas do serviço digital de fisioterapia, visando a facilitar a prestação eficaz de serviços de fisioterapia, melhorando o acesso às informaçôes sobre cuidados e o gerenciamento de recursos de saúde. No Brasil, a Resolução no 516/2020 do Conselho Federal de Fisioterapia e Terapia Ocupacional (COFFITO) corroborou as orientaçôes dos órgãos

${ }^{1}$ Mestranda em Pesquisa Clínica Aplicada à Saúde da Mulher. Fisioterapeuta do Hospital do Câncer II (HC II) do Instituto Nacional de Câncer José Alencar Gomes da Silva (INCA). Rio de Janeiro (RJ), Brasil. Orcid iD: https://orcid.org/0000-0003-0216-4669

${ }^{2}$ Doutoranda em Pesquisa Clínica Aplicada à Saúde da Mulher. Fisioterapeuta do HC II/INCA. Rio de Janeiro (RJ), Brasil. Orcid iD: https://orcid.org/0000-0001-7655-7102

${ }^{3}$ Mestre em Engenharia Biomédica. Coordenação de Pós-Graduação e Pesquisa de Engenharia (Coppe) da Universidade Federal do Rio de Janeiro (UFRJ). Fisioterapeuta do HC II/INCA. Rio de Janeiro (RJ), Brasil. Orcid iD: https://orcid.org/0000-0001-9362-4231

Endereço para correspondência: Raquel Boechat de Moura Carvalho. Rua Equador, 831 - Santo Cristo. Rio de Janeiro (RJ), Brasil. CEP 20081-250.

E-mail: raquel.boechat@inca.gov.br 
internacionais e, assim, permitiu, desde março de 2020, a teleconsulta e o telemonitoramento, evitando o contato direto e a menor exposição dos pacientes ${ }^{8}$.

\section{DESENVOLVIMENTO}

A Covid-19 apresentou oportunidades de utilizar ferramentas digitais para melhorar o acesso das pacientes, mantendo a qualidade e segurança dos serviços de fisioterapia. Na prática, é uma transformação rápida, na qual serviços passam a ser baseados em comunicaçáo (visitas eletrônicas, check-ins virtuais), além de economizar recursos escassos, incluindo equipamentos de proteção individual ${ }^{9}$.

A teleconsulta e telemonitoramento são realizados por meio de aplicativos via celular, computador ou tablet e são indicados para realização de atividades educativas, orientaçóes de atividades de autocuidado e continuidade da cinesioterapia em casos que previamente já tenham sido avaliados de modo presencial ${ }^{10,11}$. Embora existam limitações para a falta de exame físico prático com a teleconsulta, um exame virtual modificado por videochamada pode permitir um plano inicial de tratamento, por exemplo, observar a aparência, o movimento ou no autoexame sob orientação ${ }^{12}$. Antes da visita, é fundamental realizar a anamnese. Assim, medidas poderão ser tomadas remotamente a partir da avaliação clínica por videochamada e baseada no relato da paciente ${ }^{13}$.

Levando em consideração as consequências de não cuidar dessas pacientes pela incerteza da duraçáo do distanciamento social pela Covid-19, vislumbra-se a possibilidade de realizar a teleconsulta diante das principais queixas das pacientes em seguimento de Fisioterapia Oncoginecológica, para ajudar a orientar o atendimento para tratamento de complicaçóes comuns a essa clínica, como: (1) dor; (2) linfedema/síndrome pós-trombótica; (3) disfunção sexual; (4) incontinência urinária; (5) neuropatias periféricas. Além disso, existem questóes que permeiam as necessidades do tratamento oncoginecológico - mulheres que, durante o isolamento social, poderão sofrer diretamente com o aumento conflitos interpessoais, violência e ferimentos domésticos ${ }^{13}$ e o monitoramento do estado geral dessas pacientes, ainda que a distância, é muito importante ${ }^{10}$.

\section{CONCLUSÃO}

Existe um cuidado redobrado a ser tomado quanto aos critérios de prevenção e precauçôes de disseminação do novo coronavírus em pacientes com câncer, pela sua vulnerabilidade clínica e pelo maior risco de desenvolver quadros graves da Covid-19.
A fisioterapia digital por teleconsulta e telemonitoramento mostra-se um recurso promissor para a manutenção dos cuidados de pacientes com câncer ginecológico. Evitando a interrupção do acompanhamento neste momento, minimizando o comprometimento dos resultados de saúde, reduzindo as idas aos hospitais/ ambulatórios, o risco de contaminação, e favorecendo a funcionalidade dessas mulheres.

A boa aplicabilidade que tem sido observada na prática clínica da utilização desse recurso desperta interesse na apresentaçáo de um protocolo ao Comitê de Ética em Pesquisa, vislumbrando analisar os resultados que vêm sendo obtidos e, assim, melhor elucidar os benefícios da fisioterapia digital nos cuidados de mulheres com câncer ginecológico.

\section{CONTRIBUIÇÕES}

Todos os autores contribuíram substancialmente na concepção e delineamento do estudo; obtenção, análise e interpretação dos dados; redação e revisão crítica e aprovaram a versão final a ser publicada.

\section{DECLARAÇÃO DE CONFLITO DE INTERESSES}

Nada a declarar.

\section{FONTES DE FINANCIAMENTO}

Não há.

\section{REFERÊNCIAS}

1. Fauci AS, Lane HC, Redfield RR. Covid-19-navigating the uncharted [editorial]. N Engl J Med. 2020 Mar 26;382(13):1268-1269. doi: https://doi.org/10.1056/ NEJMe2002387

2. Bai Y, Yao L, Wei T, et al. Presumed asymptomatic carrier transmission of COVID-19. JAMA. 2020;323(14):14067. doi: https://doi.org/10.1001/jama.2020.2565

3. Liang W, Guan W, Chen R, et al. Cancer patients in SARS-CoV-2 infection: a nationwide analysis in China. Lancet Oncology. 2020;21(3):335-7. doi: https://doi. org/10.1016/S1470-2045(20)30096-6

4. Thuler LC, Melo AC. Sars-CoV-2/Covid-19 em pacientes com câncer. Rev Bras Cancerol. 2020;66(2):e00970. doi: https://doi.org/10.32635/2176-9745. RBC.2020v66n2.970

5. Gosain R, Abdou Y, Singh A, et al. COVID-19 and cancer: a comprehensive review. Curr Oncol Rep. 2020;22(5):53. doi: https://doi.org/10.1007/s11912-020-00934-7

6. Instituto Nacional de Câncer José Alencar Gomes da Silva [Internet]. Rio de Janeiro: INCA; [1996]. INCA contra coronavírus: Instituto toma medidas duras, mas 
necessárias, para garantir a integridade de pacientes, familiares e servidores durante a pandemia; 2020 mar 16 [acesso 2020 maio 26]. Disponível em: https://www. inca.gov.br/noticias/inca-contra-coronavirus.

7. Aquino EML, Silveira IH, Pescarini JM, et al. Medidas de distanciamento social no controle da pandemia de COVID-19: potenciais impactos e desafios no Brasil. Ciênc Saúde Coletiva. 2020;25(Supl.1):2423-25. doi: https:// doi.org/10.1590/1413-81232020256.1.10502020

8. Conselho Federal de Fisioterapia e Terapia Ocupacional (BR). Resolução no 516, de 20 de março de 2020. Dispóe sobre a suspensão temporária do Artigo 15, inciso II e Artigo 39 da Resolução COFFITO no 424/2013 e Artigo 15, inciso II e Artigo 39 da Resolução COFFITO no 425/2013 e estabelece outras providências durante o enfrentamento da crise provocada pela Pandemia do COVID-19 [Internet]. Diário Oficial da União, Brasília, DF; 2020 mar 23. Seção I, p. 184. [acesso 2020 maio 18]. Disponível em: http://www.crefito2.gov.br/ comunicados/comunicados/coffito-publicaresolucao-n516-2020-permitindo-at-3144.html.

9. World Confederation for Physical Therapy; International Network of Physiotherapy Regulatory Authorities. Report of the WCPT/INPTRA digital physical therapy practice task force [Internet]. London: WCPT/INPTRA; 2019 May 25 [cited 2020 May 18]. Available from: http://www.inptra. org/portals/0/pdfs/ReportOfTheWCPTINPTRA_ DigitalPhysicalTherapyPractice_TaskForce.pdf

10. Lee A. COVID-19 and the advancement of digital physical therapist practice and telehealth. Phys Ther. 2020 Apr 28. doi: https://doi.org/10.1093/ptj/pzaa079 Online ahead of print. PubMed PMID: 32343836.

11. Associação Brasileira de Fisioterapia em Saúde da Mulher. Recomendação geral ABRAFISM: fisioterapia por meio digital/teleconsulta e telemonitoramento na fisioterapia em saúde da mulher e uro-proctologia [Internet]. Salvador, BA: ABRAFISM; 2020 maio [acesso 2020 maio 18]. Disponível em: http://crefito7. gov.br/wp-content/uploads/2020/06/ABRAFISMRecomenda\%C3\%A7\%C3\%B5es-ABRAFISMFisioterapia-por-meio-digital.pdf

12. Eccleston C, Blyth FM, Dear BF, et al. Managing patients with chronic pain during the COVID-19 outbreak: considerations for the rapid introduction of remotely supported (eHealth) pain management services. Pain. 2020;161(5):889-93. doi: https://doi.org/10.1097/j. pain.0000000000001885

13. del Carmen MG, Rice LW, Schmeler KM. Global health perspective on gynecologic oncology. Gynecol Oncol. 2015;137(2):329-34. doi: https://doi.org/10.1016/j. ygyno.2015.03.009 